\title{
The future of power systems: Challenges, trends, and upcoming paradigms
}

\author{
João Abel Peças Lopes ${ }^{1,2}$ (1) ～André Guimarães Madureira ${ }^{1} \quad$ | Manuel Matos ${ }^{1,2}$ \\ Ricardo Jorge Bessa $^{1}$ | Vítor Monteiro ${ }^{3}$ | João Luiz Afonso ${ }^{3}$ | \\ Sérgio F. Santos $^{4}$ | João P. S. Catalão ${ }^{1,2}$ | Carlos Henggeler Antunes ${ }^{5}$ | \\ Pedro Magalhães ${ }^{6}$
}

\author{
${ }^{1}$ Institute for Systems and Computer \\ Engineering, Technology and Science, \\ Porto, Portugal \\ ${ }^{2}$ Faculty of Engineering, University of \\ Porto, Porto, Portugal \\ ${ }^{3}$ Centro ALGORITMI, University of \\ Minho, Guimarães, Portugal \\ ${ }^{4}$ Center for Mechanical and Aerospace \\ Science and Technologies, University of \\ Beira Interior, Covilhã, Portugal \\ ${ }^{5}$ INESC Coimbra and University of \\ Coimbra, Coimbra, Portugal \\ ${ }^{6}$ INESC Coimbra, Coimbra, Portugal

\section{Correspondence} \\ João Abel Peças Lopes, Institute for \\ Systems and Computer Engineering, \\ Technology and Science, Porto, Portugal. \\ Email: jpl@fe.up.pt

\section{Funding information} \\ ERDF - European Regional Development \\ Fund through the Operational Programme \\ for Competitiveness and \\ Internationalisation - COMPETE 2020 \\ Programme, and by National Funds \\ through the Portuguese funding agency, \\ FCT - Fundação para a Ciência e a \\ Tecnologia, Grant/Award Number: \\ SAICTPAC/0004/2015- POCI- \\ 01-0145-FEDER-016434
}

\begin{abstract}
The decarbonization of the economy, for which the contribution of power systems is significant, is a growing trend in Europe and in the world. In order to achieve the Paris Agreement's ambitious environmental goals, a substantial increase in the contribution of renewable sources to the energy generation mix is required. This trend brings about relevant challenges as the integration of this type of sources increases, namely in terms of the distribution system operation. In this paper, the challenges foreseen for future power systems are identified and the most effective approaches to deal with them are reviewed. The strategies include the development of Smart Grid technologies (meters, sensors, and actuators) coupled with computational intelligence that act as new sources of data, as well as the connection of distributed energy resources to distribution grids, encompassing the deployment of distributed generation and storage systems and the dissemination of electric vehicles. The impact of these changes in the distribution system as a whole is evaluated from a technical and environmental perspective. In addition, a review of management and control architectures designed for distribution systems is conducted.

This article is categorized under:

Energy Infrastructure $>$ Systems and Infrastructure

Energy Infrastructure $>$ Economics and Policy
\end{abstract}

\section{KE Y W O R D}

distributed control, management architectures, renewable generation, storage systems

\section{1 | INTRODUCTION}

Power systems have been continuously evolving since the beginning of their deployment during the late period of the Industrial Revolution. From the first commercial power plant, in Pearl Street, New York City, which featured directcurrent (DC) generators to power a New York City one square mile block, to a sprawling web of power lines across land and sea connecting often distant power plants generating alternating current (AC) electricity and numerous points of use, significant progress has been made in power system technology with regard to efficiency, quality, safety, and 
reliability (Hughes, 1993). This evolution enabled power systems to assume an increasing importance in peoples' lives and the world economy, to an extent that has made reliable power systems a distinctive feature of modern societies, which partly explains the difficulty in transforming them to take advantage of new opportunities and tackle further modern day challenges (Smil, 2004).

These challenges for power systems largely emanate from the need to deal with the climate crisis and promote a more efficient and reliable use of existing resources and infrastructures (Strbac, 2008). The Paris Agreement, according to which parties are expected to contribute in order to ensure that the increase in global average temperature above preindustrial levels remains below the $2^{\circ} \mathrm{C}$ threshold and pursue additional efforts to prevent it from exceeding $1.5^{\circ} \mathrm{C}$, implies parties have to undertake swift actions to reduce greenhouse gas (GHG) emissions. As an example, this commitment prompted the European Commission to propose a 40\% cut in GHG emissions relative to 1990 levels by 2030, among other measures (European Commission, 2014; Poudineh, Peng, \& Mirnezami, 2017). Policies such as these have direct consequences on power systems since electricity generation is responsible for a substantial share of global emissions (up to $42 \%$ in 2013), predominantly due to fossil fuel-fired power stations following a study from the International Energy Agency (IEA, 2015). Replacing these technologies with more environmental-friendly and sustainable ones without compromising the range and quality of services originally provided is by itself a formidable task, since this implies integrating a significant amount of (mostly nondispatchable) renewable generation technologies with limited (or no) inertia, such as wind turbine generators and photovoltaic (PV) panels, the adoption of energy storage solutions, and the expansion of transmission and distribution networks. However, this already onerous task will likely be made more challenging by the additional demand predicted due to the trends for the adoption of electrical heaters and vehicles, as both heat and transport energy end users also move away from fossil fuels (Heinen, Mancarella, O'Dwyer, \& O'Malley, 2018; Poudineh et al., 2017). For example, Ruhnau, Bannik, Otten, Praktiknjo, and Robinius (2019) predict that electricity consumption in Germany, which reached 570 TWh in 2016, could more than double by 2050 due to heat and transport $(+400-800 \mathrm{TWh})$. As such, the main challenge is in significantly changing power systems without compromising the safety and reliability standards achieved with conventional power systems while the demand for electricity grows considerably.

In order to ensure national economies are progressively decarbonized, power system generation portfolios will also need to change over time to approach or become $100 \%$ renewable. Large-scale (wind and PV) renewable power plants will need to be commissioned together with a massive deployment of small-scale distributed generation (DG) using primary renewable power sources. This is expected to happen by 2050, at least in Europe where a very ambitious energy policy plan is underway. The big changes from a structural and market point of view will take place mainly between 2030 and 2050.

Since electricity demand is expected to grow in many countries, for different reasons, end users can play a positive role in addressing these challenges, namely through demand side management (DSM). DSM has been traditionally concerned with promoting the efficient use of existing power system infrastructure including fossil fuel-fired power plants (Eto, 1996; Garcia, 1987; Gellings, 1985; Gellings \& Smith, 1989; Lotfi, Monteiro, Shafie-khah, \& Catalão, 2018). The basic thrust of DSM is to make consumers aware of state of the grid vis-à-vis supply availability and penalize those burdening the system when it is most vulnerable, thus rewarding or inducing a more rational use of available resources. Achieving these goals becomes more tenable with the developments in information and communication technologies (ICT), since they enable bidirectional communications between grid operators and end users, namely to relay dynamic electricity prices, the availability of local generation resources, the requests of load flexibility, and also greatly facilitating load scheduling and consequently the integration of DG (Siano, 2014; Strbac, 2008). Hence, these technologies not only allow for reductions of peak demand but also the provision of additional (ancillary) services, which could contribute to maintain or improve quality of service standards in power systems, namely by taking advantage of the amount of data generated to make an integrated optimization of all energy resources. On the other hand, this prospect raises concerns about privacy, potential intrusions and resiliency to communication failures, which need to be addressed (Deng, Yang, Chow, \& Chen, 2015; McKenna, Richardson, \& Thomson, 2012).

Another important concern relates to the impact of bidirectional energy flows in modern power systems. Power systems have traditionally relied on unidirectional flows to keep system operation simple and economic; however, the presence in distribution systems of large numbers of consumers also capable of producing electricity that can cause bidirectional flows in systems not designed with this feature in mind and complicate the design of protection systems and other components essential to maintain high reliability levels (De Carne, Buticchi, Zou, \& Liserre, 2017). DG could allow for islanded operation of microgrids, thus increasing their resilience to power outages and other events, but this also introduces safety hazards for maintenance personnel and equipment (Bollen \& Hassan, 2011). Hence, in addition 
to properly sized components, such as transformers and power lines, such changes require adequate protection systems and system architectures to maintain the same standards currently enjoyed in modern power systems (Bollen \& Hassan, 2011; Papaspiliotopoulos, Korres, Kleftakis, \& Hatziargyriou, 2015; Schneider et al., 2019).

In addition, issues such as variability and uncertainty of both demand and renewable-based generation, despite their increased relevance today, are recurring features of power systems that have always been dealt with to ensure adequate supply reliability (Chandler, 2011). Safety and environmental standards have also improved over time, namely regarding concerns over human health due to pollution and other hazards. Hence, the challenge lies then on how to adequately address these issues in an integrated manner while considering their specific characteristics and making use of the most effective and efficient solutions at hand.

\section{2 | IDENTIFICATION OF FUTURE CHALLENGES TO POWER SYSTEMS}

In this section, some of the most relevant challenges for future power systems are outlined. Each challenge is summarily described to set the basis for the definition of dedicated management and control architectures and advanced functionalities that can be used for the distribution networks of the future. These challenges are summarized in Figure 1.

\section{1 | Connection of small distributed energy resources (DERs) to distribution grids}

Future power grids should have a set of new features that will be necessary to satisfy the needs of the grid operators and the consumers, aiming at increasing the resilience of the power grids considering the integration of new emergent technologies (electric mobility, microgeneration systems, and energy storage systems). DG, where microgeneration at the customer's premises can be included, together with dynamic response from loads and distributed storage units, with

FI G URE 1 Future challenges to power systems

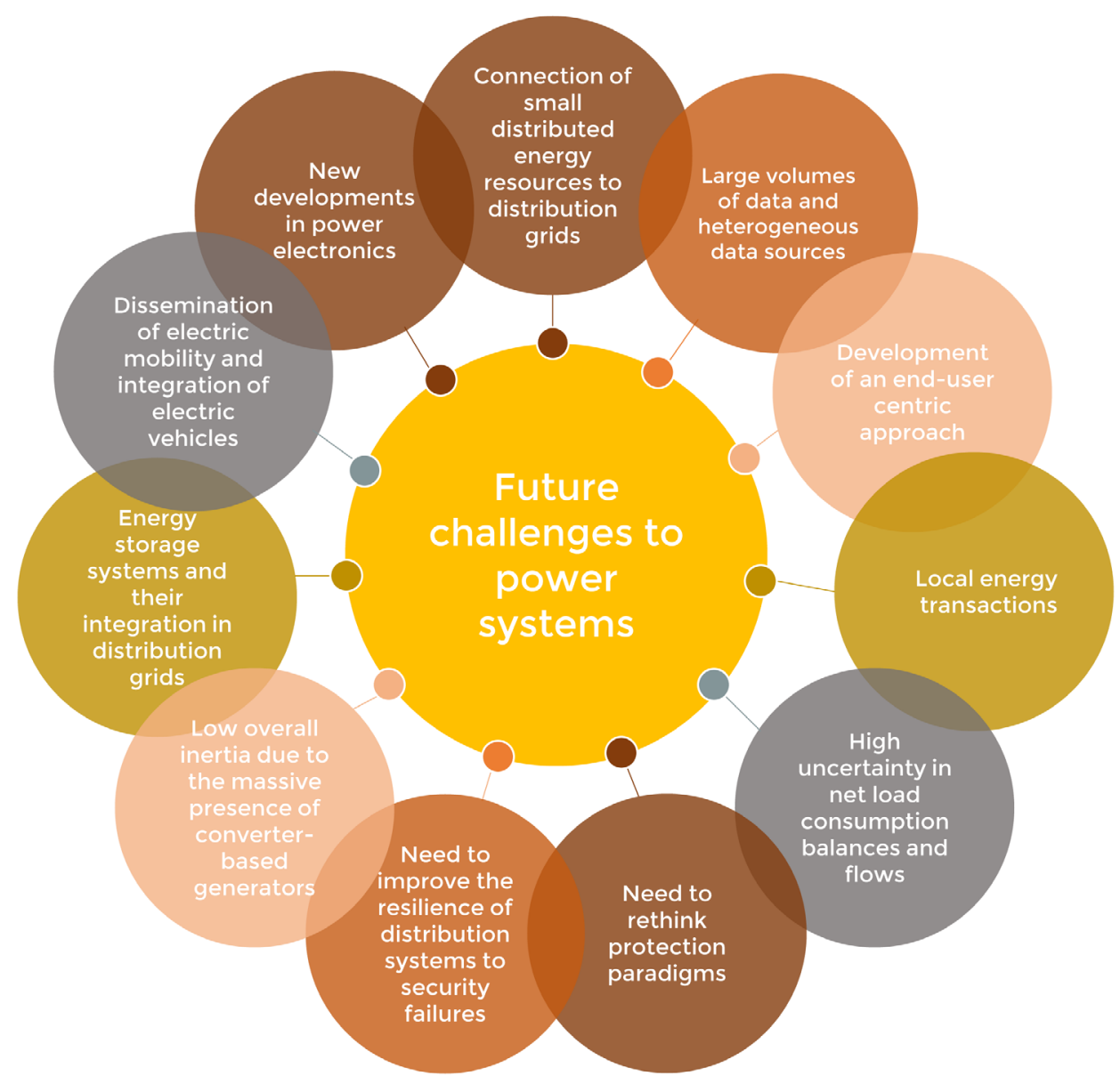


batteries from electric vehicles (EVs) also operating in Vehicle-to-Grid (V2G) mode, form the plethora of the DERs. With the integration of these emergent technologies, all of them establishing (unidirectional or bidirectional) communication with the power grid, a reliable control strategy will be required to manage the power transactions and the network control in real time, especially considering the dynamic and distributed integration of electric mobility systems and demand response programs in face of dynamic tariffs.

The large-scale integration of DG will require the adoption of new concepts such as renewable energy communities, and an interaction with traders/aggregators that will serve as interfacing entities that can bid energy and services at local/regional levels or in the wholesale market (as defined in the new EU energy directive 2018/2001).

\section{2 | Large volumes of data and heterogeneous data sources}

Large amounts of data are being generated at different segments of the power sector as it is transformed by the ongoing digital revolution. These data has a potential value for power companies, grid operators, and end users, and can be exploited using big data algorithms for different purposes as power supply and demand forecasting, state estimation and grid control as well as to foster participation in electricity markets (Bessa, 2018). The knowledge extracted from these data is thus expected to positively impact indicators relating to operation and maintenance costs, investment postponement, and quality of service. Furthermore, big data processing and mining are prompting the emergence of new business models (e.g., trading companies exploiting large datasets to better understand the behavior of their clients) and boosting the offer of new (customized) energy services to consumers.

Moreover, in future power systems, it is expected that a significant part of the electrical appliances will have access in real time to some variables of power grids (e.g., system frequency). In addition to the necessary control of the electrical appliances, such parameters can be useful for supervision algorithms to evaluate if the control signals are consistent with the real operation of the power grids. The definition of mechanisms and supervision algorithms will be extremely important to prevent damages in the operation of the systems in case of failures in the command and management systems or in cases of attack or intrusion in the communication networks, having as main objective the efficient and sustainable utilization of electrical energy, both for consumers and electricity grid operators.

\section{3 | Development of an end-user centric approach}

In the future, an efficient and sustainable utilization of the electrical energy in an end-user centric approach is expected, ensuring the dynamic involvement of consumers along with the awareness of the needs of the power grid. For such purpose, the development of increasingly efficient, autonomous, and resilient technological solutions is required, allowing to perform the interface between the different systems coupled to the power grids, as well as communication systems enabling end-user involvement in a dynamic way. These communication systems will allow defining periods of utilization for the electrical appliances according to power grid operators and end-users convenience, also integrating monitoring systems, to know in real time the energy transactions (for instance, when the end user is a prosumer) and minimize the inefficient and unnecessary use of the electrical appliances. Moreover, by controlling such periods of operation of the electrical appliances, it will be possible to define control strategies to optimize the utilization of the power grid, for example, using load shift, load shedding, and thermostatic load reparameterization mechanisms, which encompasses the concepts of peak shaving and load leveling. These mechanisms aim at changing consumption patterns to better match demand and supply (i.e., within a demand response paradigm). In Talari et al. (2017), a literature review is carried out on this subject, with emphasis on the use of internet-of-things (IoT) technologies for Smart Cities. Machine-to-machine (M2M) supervisory control and data acquisition (SCADA) systems are examples of such solutions.

\subsection{Local energy transactions}

With the expected empowerment of prosumers and emergent technologies (such as blockchain) that enable local trading of renewable energy and flexibility, the design of optimization models and new business models to enable prosumers peer-to-peer (P2P) trading is fundamental. 
In the literature, three market mechanisms are defined for prosumers with local renewable energy sources (RES) and willing to trade demand-side flexibility: (a) P2P trading to buy and sell energy services; (b) provide services to microgrids within utility grids; and (c) supply services to an isolated microgrid (Parag \& Sovacool, 2016). Real-world examples of P2P trading platforms are Vandebron (Netherlands) and Piclo (UK) which offer online platforms for RES producers. In turn, the Horizon 2020 European Project P2P-SmartTest sought to explore energy trading between peers, including small-scale DERs, and investigate hierarchical structures within distribution grids (Zhang, Wu, Cheng, Zhou, \& Long, 2016). In this context, a dynamical internal pricing model can be formulated in a distributed iterative way to support PV energy sharing among neighboring prosumers (Liu et al., 2017).

This P2P concept is not new and was already discussed in Wu and Varaiya (1999). However, two emergent technologies are creating opportunities to boost P2P markets for energy and flexibility: distributed optimization and blockchain. These technologies have been already explored to create a distributed learning framework for wind power forecasting (Cavalcante, Bessa, Reis, \& Browell, 2017) and distributed optimal power flow (OPF) algorithms in smart grids (Christakou, Tomozei, Le Boudec, \& Paolone, 2017). Blockchain uses decentralized ledger books to record all transaction data and does not require intermediaries or a central entity. As such, this technology facilitates the creation of decentralized energy markets in which producers and consumers can sign energy contracts directly without supervision from authorities.

\subsection{High uncertainty in net load consumption balances and flows}

The power systems of many countries have been able accommodate rising shares of RES and this trend is set to continue. However, this trend poses significant challenges to the existing power systems due to the difficulty in coping with the inherent time-varying nature of RES when these resources dominate generation portfolios (Chandler, 2011). Among other technical challenges, frequency and voltage control can be impacted by RES-prompted mismatches between supply and demand. In general, a positive correlation exists between the share of variable power sources and the power system flexibility needs. Flexibility, in this setting, concerns the extent to which such systems are able to manage unpredicted operating conditions, namely those induced by the intrinsic load and distributed RES generation variability (Villar, Bessa, \& Matos, 2018). Hence, without adequate flexibility mechanisms, it becomes increasingly harder to cope with mismatches between generation and demand stemming from their natural variations in real time as the share of RES increases. Moreover, it is also necessary to develop new grid management tools that include information from uncertainties associated with load and local generation changes, such as stochastic OPF (Soares, Bessa, Pinson, \& Morais, 2018).

\section{6 | Need to rethink protection paradigms}

Protection systems in current distribution networks assume a unidirectional flow of current, from the upstream substation to the loads. In the presence of DG units injecting power in the network, a different situation arises. In fact, faults may be fed from other sources than just from the upstream substation, causing short circuit currents to flow in certain branches that are different from those that would flow for an equivalent short circuit with only one upstream power source. Furthermore, short circuits are also potentially fed from distributed power injection points. Fault currents detection and measurement become jeopardized and existent protection devices may not react to ensure appropriate fault isolation. Technical solutions have to be found for these problems, which may require significant investments. The network upgrading measures involved in the implementation of the smart grid paradigm will have to coherently include the protection system upgrading which, in turn, will have the potential to help implementing fully the microgrid concept as well.

\subsection{Need to improve the resilience of distribution systems to security failures}

Distribution System Operators (DSOs) should provide reliable services to consumers by planning system infrastructures and operation according to forecasts and grid conditions. However, when abnormal and/or catastrophic events occur significant service interruptions are likely. With the proliferation of small-scale DG and energy storage 
systems, as well as the development of innovative grid technologies such as the smart transformer (Huber \& Kolar, 2017), it is expected that, in some cases, the microgrid concept can be exploited as part of a resiliency plan to minimize the interruption of power to critical loads. This could involve the creation of electrical islands using local generation resources to feed at least part of the load, and exploiting storage units to keep the balance between load and generation.

Power systems are increasingly facing more disruptions from extreme weather events such as hurricanes, large fires, floods, and so on while societies remain, and are set to become even more, extremely dependent on electricity. Resilience is therefore an ever increasing requirement that goes further than N-1 security. This involves the capability of islanding operation of parts of the distribution grid and the development of more effective black start procedures such as the ones that exploit bottom-up strategies using DER control capabilities (Moreira, Resende, \& Lopes, 2007).

\subsection{Low overall inertia due to massive presence of converter-based generators}

There is a massive ongoing transformation within the power system, including the control of these systems. The traditional power system has relied on a large number of synchronous generators to provide voltage and frequency control. The penetration of new forms of renewable energy generation generators are convertors based generators is rapidly increasing and in future power systems with 100\% RES generation, it the need for power electronics interfaced generation systems to provide active contributions toward grid stability and security will become essential to the operation of these power systems (Ackermann et al., 2017; Milano, Dörfler, Hug, Hill, \& Verbič, 2018). For instance, during some hours in March 2016, 100\% of the load in Portugal could be covered by renewable energies (mainly wind power and hydropower) during four consecutive days. In this case, a large amount of hydrosynchronous generation was also connected to the grid providing frequency control services. The energy transition policy that will be followed in Europe will increase largely wind and PV power, replacing conventional thermal generation. This policy will require services such as frequency containment reserve or the provision of synthetic inertia in order for the system to operate in a robust and secure manner.

In addition, requirements for grid connections, which typically are appropriate for transmission system stability (including capabilities for active power frequency response by power generating modules in limited frequency sensitive mode at overfrequency-LFSM-O, as referred in the network code on requirements for generators of ENTSO-E ${ }^{1}$ ) not always have a positive effect at the distribution level. Likewise, solutions which work at the distribution level (such as local voltage control of DG units) are not as effective for the transmission level. Because of this, it is crucial to determine the impact of various support services at the varying voltage levels and it is also important to consider any possible interactions that might occur between the different services. Thus, a framework of a distribution system operator/transmission system operator (DSO-TSO) interaction will be essential to understand the full impact of the large-scale integration of RES on the distribution grid.

\section{9 | Energy storage systems and their integration in distribution grids}

Nowadays, the concept of energy storage systems embraces several technologies with different states of maturity, including pumped hydro, electrochemical batteries, compressed air systems, and flywheels. The management of such diversity of technologies represents an important challenge for power grids, mainly taking into account the integration of renewables into the power grid concerning the voltage levels (high voltage- $\mathrm{HV}$, medium voltage- $\mathrm{MV}$, and LVlow voltage ), the management flexibility needed to deal with uncertainties, and the necessary decentralization of the distribution system control. It is important to note that, whenever possible, energy storage systems should be installed near RES and near end users, allowing to mitigate the problems related to the variability of the energy produced from RES. Moreover, with this strategy, it is possible to use unified converters for interfacing renewables and storage systems with the power grid. As experimentally validated in Goli and Shireen (2014); Monteiro, Pinto, and Afonso (2018); and Hamilton et al. (2010) with unified converters, a common DC-link is used as interface for renewables and storage systems, allowing a single interface with the power grid. Therefore, compared with conventional solutions, it is possible to reduce the need for two power converters to interface the power grid, also allowing improving the efficiency. In addition to the aforementioned storage systems, power to gas systems can represent an important contribution for the expected change of paradigm in the future power grid (Götz et al., 2016), once such solution (assuming that Hydrogen 
is obtained from renewable sources) can allow increasing storage capabilities. In this context, heating storage systems can also be considered as an important contribution due to their flexibility in countries where intensive utilization of heating systems is required (Buttler, Koltun, Wolf, \& Spliethoff, 2015).

\subsection{0 | Dissemination of electric mobility and integration of EVs}

A short presentation of the main trends and challenges in road transportation electrification can be found in Cazzola, Gorner, Munuera, Schuitmaker, and Maroney (2017) and Pereirinha et al. (2018). The sales of electric light vehicles exceeded 1.2 million in 2017 and in addition, 500,000 heavy plug-in vehicles were sold bringing the total of EVs to 3.2 million. The sales of EVs are expected to increase significantly with $24 \%$ (54\%) of new car sales and $7 \%$ (33\%) of total vehicles on the road will be electric and this growth in EVs places new and important challenges to the grid. These challenges are made harder by the tendency to increase both the capacity (with some passenger vehicles exceeding $100 \mathrm{kWh}$ and electric buses and luxury vehicles exceeding $350 \mathrm{kWh}$ ) and the rate of charge (such as ultrafast charging) (Carrilero et al., 2017). These vehicles can also have a positive contribution as they can be used to increase the flexibility of the grid through grid-to-vehicle (G2V) and V2G operation modes. As a contribution for the electric mobility dissemination, policy measures including financial support schemes are necessary to foster the adoption and development of this new paradigm. However, such financial incentives should be adaptable enough to accommodate the large-scale integration of EVs, as well as the new predictable technologies (for instance, new materials and technologies for batteries, new power ratings for EV battery chargers, EV performance, and new operation modes for the EV besides G2V and V2G). In Monteiro, Pinto, and Afonso (2015), some new operation modes for the EV are presented: (a) Home-tovehicle $(\mathrm{H} 2 \mathrm{~V})$, which consists in controlling the EV operating power as a function of the appliances operating in the smart home (this mode can be linked with the G2V and V2G modes, allowing charging or discharging the battery according to the appliances); (b) vehicle-to-home (V2H), which consists in using the EV as a voltage source (e.g., in camping) or as an uninterruptible power supply (UPS) in a smart home context (only applied during power outages); and (c) vehicle-for-grid (V4G), which consists in using only the EV battery charging system for providing services for the grid, that is, the battery is not used to exchange reactive energy with the power grid, representing an attractive mode for smart grids and smart homes (this operation mode contributes to the smart grid or smart home, since it can produce reactive power, or it can compensate current harmonic caused by appliances).

Especially important is the EV charger when the EV is used in these different modes. Chargers offer dynamic and distributed integration in the power grid. An EV can be plugged in for charging at one location (G2V) and then driven to a different location where the EV could be used to deliver energy back into the grid (V2G). To account for the increasing charging power, some charging locations are already making use of on-site energy storage in order to help smooth out the peak power demanded from the grid. This offers up more modes of operation as the charging station can now participate in energy management actions when the local storage is charged from the grid (G2LS) and also when the local storage is discharging into the grid (LS2G).

The large-scale integration of EVs into the power grid will bring relevant environmental benefits, especially if the required power is produced from renewables. Otherwise, if the necessary power for charging the battery is provided by non-RES, a well-to-wheels analysis concerning the emissions is fundamental to compare the emissions of EVs with the emissions of conventional vehicles with internal combustion engines (Elgowainy et al., 2010; Milberg \& Schlenker, 2010). Otherwise, the total well-to-wheels emissions can, in some cases, be greater when compared with the emissions of conventional vehicles with internal combustion engines. It is important to note that the EV has low GHG emissions at the usage level, but the life cycle analysis should consider the energy source used for the battery charging process (Hall \& Lutsey, 2018). In this context, new possibilities arise for the flexible operation of EVs into power grids, since integrated systems with RES can be implemented, allowing, for instance, to charge the EV batteries directly from renewables without the power grid as intermediary. It is also important to highlight that these know-hows that can be viable not only for EVs (i.e., pure plug-in battery EVs), but also for other technologies framed with e-mobility as hybrid vehicles, e-bicycles, and electrical railway systems.

Another challenge is aggregating thousands of EVs and be able to control the flow of energy in G2V and V2G as if it was a single power station that can participate in the market, according to the requirements of the network and the interest of vehicle owners. 


\subsection{New developments in power electronics}

Aiming the challenges and new paradigms of future power systems, it will be necessary to direct efforts for working areas in the scope of power electronics, used for the development of technological solutions for power grids, including advanced control algorithms focusing on the optimization of the entire system. In this context, new developments of power electronics are expected for the future power grids, mainly in terms of: (a) smart transformer; (b) bidirectional wireless battery charger for electric mobility applications; and (c) unified topologies with concerns of power quality.

Considering the advances of power electronics technologies, new perspectives are emerging toward power control in a smart grid. In this scenario, the smart transformer represents a suitable support for interfacing different systems with the smart grid and compete with the traditional low-frequency transformer, bringing additional control features that will improve grid operation. Since the smart transformer is based on power electronics, due to the new advances of power semiconductors and magnetic materials, it is possible to improve the efficiency of the smart transformer, for the entire spectrum of operation and almost independently of the type of load (linear or nonlinear) (Costa, De Carne, Buticchi, \& Liserre, 2017; Ko, Chub, Costa, Andresen, \& Liserre, 2018; She, Huang, \& Burgos, 2013). The efficiency of the traditional low-frequency transformer is load dependent. Moreover, with the smart transformer, it is possible to ensure high levels of power quality (even with the interface of renewables and energy storage systems through the DC-link interface). Therefore, the current in the grid side is controlled independently of the occurrence of voltage problems (for instance, a power grid voltage with high harmonic distortion), and the load-side voltage is controlled independently of the loads type (linear or nonlinear loads).

Concerning electric mobility, it can be foreseen that the introduction of charging systems with innovative topologies and operation modes, contributing for sustainable growth of the transportation sector. Therefore, charging systems based on wireless power transfer technology will be a reality, where the bidirectional operation (G2V and V2G) will be a key challenge in terms of power electronics topology.

The use of nonlinear loads, which contribute to accentuate the power quality problems, is becoming increasingly common. Some examples of nonlinear loads are conventional electrical appliances in the residential sector, such as computers, printers, televisions, light emitting diode lights, refrigerators, and washing machines, and loads in the industrial sector, such as variable speed drivers for motors.

Traditionally, active power filters are employed as a contribution to solving such problems. However, with small modifications in terms of the topology of the active power filter, it can also be employed for simultaneously interface other technologies in a smart grid. Therefore, the development of power electronics solutions for implementing unified topologies is expected, operating as active power filters at the AC grid side, as well as for interfacing renewables (e.g., PV solar panels) and energy storage system (e.g., batteries) at the DC side.

\section{3 | MANAGEMENT AND CONTROL ARCHITECTURES}

In this section, an overview of the most relevant types of control architectures for distribution systems is presented. A comprehensive literature review on management and control architectures was conducted in Matos et al. (2016). According to these authors, architectures range from traditional centralized architectures to hierarchical and decentralized solutions. These different architectures and their characteristics are described below.

\section{1 | Centralized architectures}

The traditional organization of power systems inherently implies a centralized architecture comprising three distinct levels: generation, transmission, and distribution. At the top level, bulk generation based on fossil fuels, nuclear or large hydropower stations produce the electrical energy that is conveyed first through long distances using HV or extra high voltage (EHV) transmission lines, and then using lower voltage levels through distribution networks to the end users.

The advent of DG is essentially altering this paradigm by having power injections directly in the distribution network. As long as only small amounts of distributed energy flows occur, a traditional fit-and-forget approach can be suitable and no fundamental changes need to be introduced; however, as the integration levels of DER (and renewablebased DG in particular) grow, centralized solutions are not able to cope with these new phenomena. 


\section{2 | Hierarchical architectures}

With the integration of DER in distribution networks, particularly DG based on renewable sources, in different voltage levels from HV to LV, more complex architectures are required to be able to operate the global system. This is necessary if a real active network management scheme is to be implemented and there is the intention to take advantage of the presence of these types of resources for control and management purposes.

The microgrid concept developed in the EU project "Large Scale Integration of Microgeneration to Low Voltage Grids" (MICROGRIDS) presents a hierarchical architecture for the control of LV distribution systems that include multiple energy resources-thermal and electrical loads and microgeneration systems in a small geographical area (Lopes, Madureira, \& Moreira, 2013). In order to promote an effective control of this type of system, storage devices and advanced network control systems are required.

The microgrid concept implies that a microgrid central controller (MGCC) is to be installed at MV/LV secondary substation level. This controller is dedicated to ensuring a safe operation of the microgrid system by including a set of control functionalities and serves as an interface to the main distribution grid and the central distribution management system (DMS). It also relies on a set of distributed controllers located at the customers' premises, namely microsource controllers (MCs) to interface small microgeneration units or storage devices and load controllers (LCs) that interface electrical loads. The MGCC is able to send set points to individual controllers (either MCs and LCs) in order to control individual resources. The microgrid architecture is shown in Figure 2.

This architecture was further extended in the EU project "Advanced Architectures and Control Concepts for More MicroGrids" (MORE MICROGRIDS). A multimicrogrid system is defined as a higher level control layer at the MV level that consists of a set of microgrids and DG units connected to the MV grid on different feeders. In this context, DG sources, microgrids, and controllable loads are considered as active cells (Lopes et al., 2013). Similar to what happens in a microgrid, a multimicrogrid system has a central controller dubbed central autonomous management controller (CAMC) located at the MV side of the HV/MV primary substation being responsible for managing the MV network located downstream. The CAMC is expected to encompass some of the functions typically assigned to a DMS and become responsible for interfacing the multimicrogrid system with the central systems (DMS) and lower level controllers (MGCCs) and remote terminal units (RTUs). For example, it is responsible for controlling the resources connected

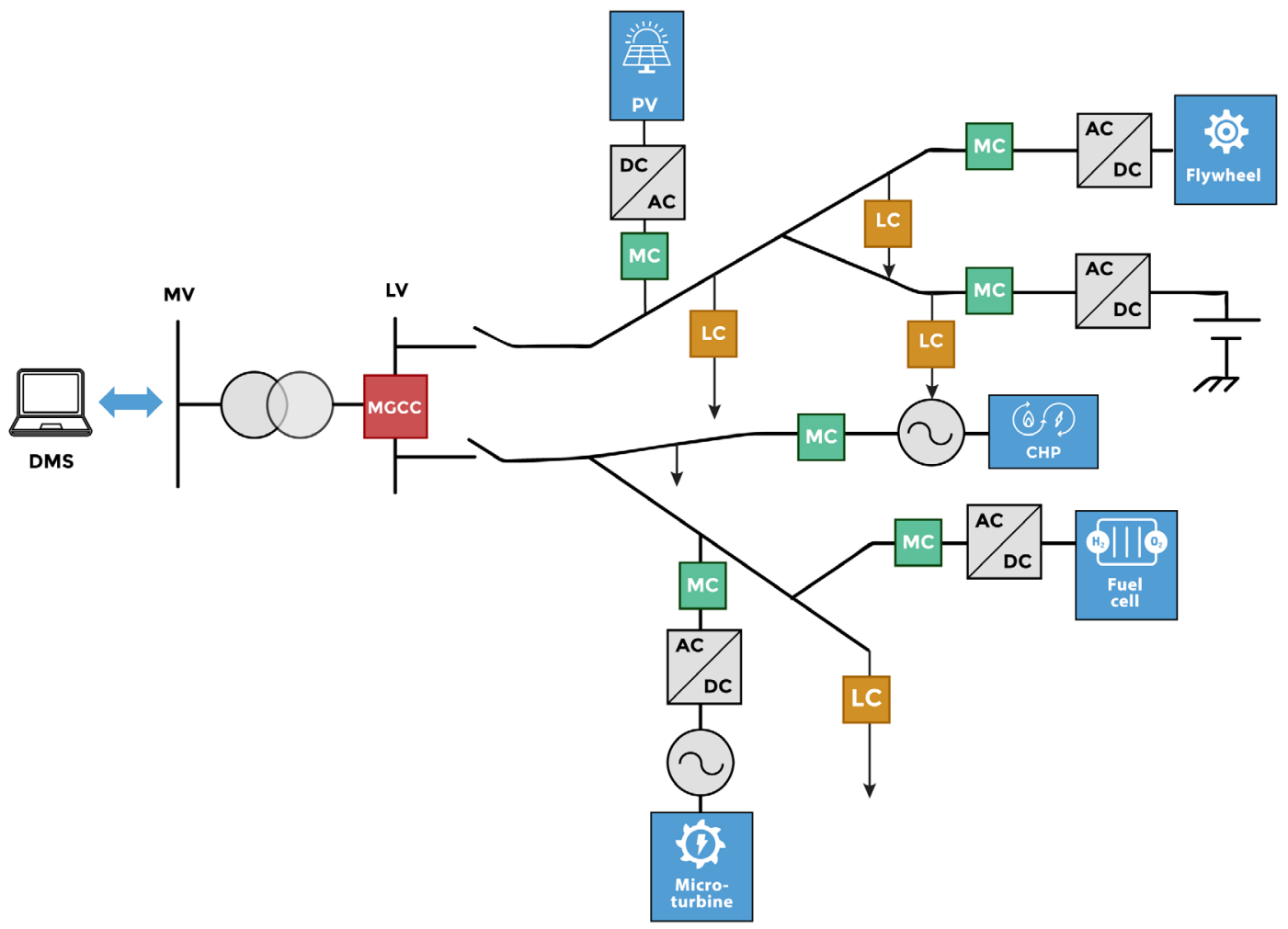

F I G U RE 2 MicroGrid architecture. (Reprinted with permission from (Lopes et al. (2013). Copyright 2013, Wiley) 


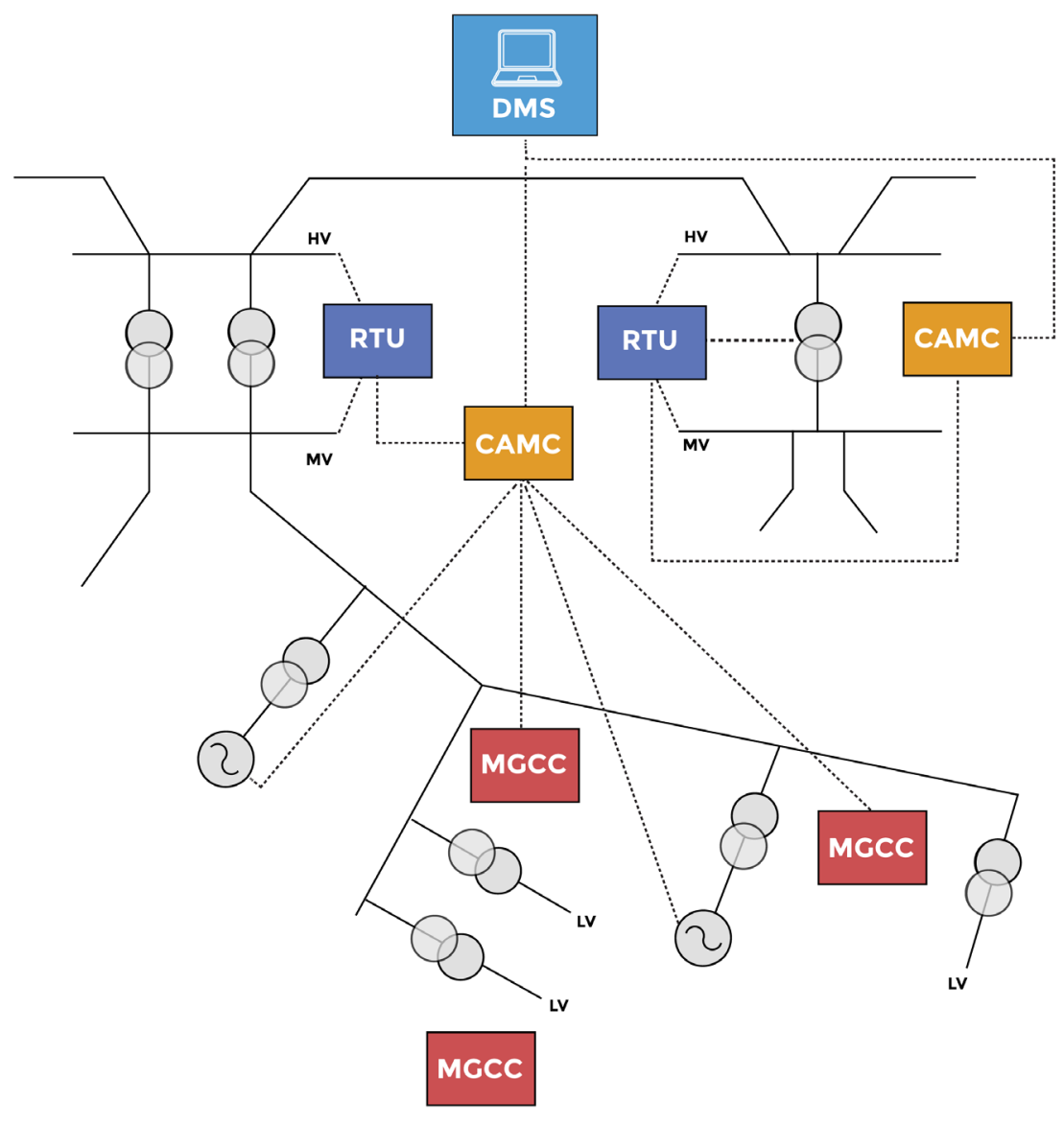

F I G U RE 3 Multimicrogrid architecture. (Reprinted with permission from Lopes et al. (2013). Copyright 2013, Wiley)

directly to the MV network, namely capacitor banks or On Load Tap Changing (OLTC) transformers (for instance, for voltage control purposes), but also other entities such as DG units, large industrial loads, and microgrids in a coordinated way. The multimicrogrid architecture is shown in Figure 3.

The flagship project for smart grids by EDP Distribuição, the Portuguese DSO-INOVGRID (Cunha, Reis, \& Lopes, 2008), which defined a control and management architecture for the distribution network, was largely inspired by the developments made in these two projects.

A number of demonstration projects implemented and tested some of these concepts in real-life demonstrators, such as the FP7 EU project GRID4EU ("Large-Scale Demonstration of Advanced Smart GRID Solutions with wide Replication and Scalability Potential for EUROPE") and its French demonstrator-Nice Grid. ${ }^{2}$

The GRID4EU project comprised six demonstrators with different boundary conditions: Sweden (Vattenfall) dedicated to monitoring an LV network through an advanced metering infrastructure and smart devices in secondary substations; France (ERDF) on optimizing PV integration in LV networks based on load and PV forecasting, controllable loads, storage devices, islanding, and consumer participation; Spain (IBERDROLA) aiming at the improving the automation of MV and LV networks as well as increasing the awareness of consumers regarding their consumption and status of the grid; Germany (RWE) dedicated to improving the monitoring and control of MV networks through the use of MAS; Czech Republic (CEZ DISTRIBUCE) dedicated to the automation of MV and LV networks including managing EV charging and islanding; and Italy (Enel) on control systems able to increase the hosting capacity in order to increase the integration of DER in MV grids.

The French demonstrator (Nice Grid) that run from 2012 to 2016 was developed in the city of Carros, on the territory of the Metropolis Nice Côte d'Azur which had significant renewable capacity, including PV (Foggia et al., 2014; Lannez et al., 2013).

The following objectives were pursued:

- Optimize the operation of a distribution network by integrating renewable energy from PV panels and using battery storage at strategic points of the network and at customer premises; 
- Reduce peak consumption at the city level by combining consumer's demand and storage;

- Study and test the operation of an autonomous consumer area, isolated from the main network and with its own means of PV generation and storage;

- Give the consumer an active role through by promoting a balance between generation, consumption, and storage"prosumer."

Other EU projects such as "Distributed Renewable resources Exploitation in electric grids through Advanced heterarchical Management" (DREAM) proposed alternative approaches such as an heterarchical management. This is basically an evolving hierarchy that evolves and adapts to different topologies and operational constraints and considers different operating modes. It aims at ensuring full local autonomy where there is potentially no need to provide global information for most of the actors involved (Caire, Lisanti, \& Vanet, 2014).

\subsection{Decentralized architectures}

Purely decentralized architectures ensure that information is compartmentalized and that global information is not required by intervening actors. One common alternative is using multiagent systems (MAS), which are based on artificial intelligence methods that resort to agent-based technology. This approach is usually suitable for complex problems where individual agents are called to find a solution to a global problem either through cooperation or competition.

In Khazaei and Nguyen (2017), a distributed control architecture is presented for battery energy storage systems (BESSs) using a MAS framework. The active/reactive power sharing, the frequency/voltage, and the energy of BESSs are synchronized by exchanging local information with a few other neighboring BESSs. Two consensus algorithms, leaderless and leader-follower, have been proposed in this context.

In Zheng, Hill, and Dong (2017), a multiagent approach for the optimal allocation of energy storage systems is proposed for multiple agents in a distribution system. It uses a game-theoretic approach, where agents reach a Nash equilibrium for BESS installation. The results obtained prove the economic feasibility and convergence of the method proposed.

Luo et al. (2016) propose a framework for cooperative control aiming at coordinating several microgrids. The framework is based on MAS and aims at encouraging resource sharing among different autonomous microgrids and solves energy imbalances by forming the microgrid coalition self-adaptively. The proposed control framework is implemented in Java Agent Development Framework (JADE).

Rokrok, Shafie-khah, Siano, and Catalão (2017) propose a decentralized MAS-based approach for LV-microgrid restoration in which a control architecture of a microgrid based on MAS is employed to determine the optimal microgrid operations management and power delivery as well as to embed distributed intelligence into the microgrid.

A novel MAS-based model and the optimal management of a microgrid integrated with RES for distribution systems is proposed in Khan, Wang, Xiong, and Ma (2018). The Java-based development tool JADE is used in the implementation of the MAS to deliver powerful errands implementation with conformation model and P2P mediator interface based on asynchronous interaction values. To assist the load demand, electrical energy can be generated by using solar $\mathrm{PV}$, small wind turbines, and fuel cells.

In Klaimi, Rahim-Amoud, Merghem-Boulahia, and Jrad (2018), JADE is also used to implement a MAS highlighting the effects of power loss on the energy cost in an electrical system and a novel approach is developed to help the storage system meeting consumers' daily demands.

In Shi and Yang (2018), the distributed optimization problem over MAS networks is considered. Different techniques, including the factorization of weighted Laplacian and the spectral decomposition technique, are used in order to attest the linear convergence of the augmented Lagrange algorithm, that can be used to solve power system problems.

Sakurama and Miura (2017) address a distributed constraint optimization problem on networked MAS. A distributed algorithm based on the Lagrangian method is proposed, where a new update law of the Lagrangian multiplier is designed, which enables each agent to estimate the value of the Lagrangian multiplier in a distributed manner. A necessary and sufficient condition is then derived so that the optimization problem can be solved in a distributed manner over a graph. The proposed method is then applied to control the power grid through distributed pricing to maintain the balance between supply and demand. 
Khan et al. (2019) and (Khan and Wang (2017) present extensive reviews on optimization and control for microgrids using MAS.

A new and upcoming paradigm is the IoT, or more specifically the internet of energy (IoE). According to this paradigm, devices located at different layers of the distribution grid (such as DER, OLTC, or residential loads) have embedded intelligence, including control algorithms, as well as internet-based communication capabilities.

\section{4 | Hybrid architectures}

Alternative architectures include hybrid solutions combining the benefits of hierarchical and distributed solutions. In the European project "Smart Distribution System OperaTion for mAximizing the INtegration of RenewABLE Generation" (SuSTAINABLE), an architecture is proposed with four main layers with a degree of autonomy (Madureira et al., 2015):

- An upper level corresponding to the SCADA/DMS managing the whole distribution grid from the HV level.

- An intermediate control level with a smart substation controller (SSC) that is able to manage the MV grid, including DG, controllable loads (CLs) and storage systems (STORE MV) at the MV level together with OLTC transformers and banks of capacitors (CAP MV) connected to the MV.

- A lower level where the LV grid is managed using a distribution transformer controller (DTC), controlling MV/LV OLTC transformers and storage devices at the LV side of the distribution transformer (STORE DT).

- A field level at the LV customers' premises, interfaced by a smart meter (energy box-EB) and managed by a Home Energy Management System (HEMS) that controls microgeneration (uG), controllable loads (CL), and storage systems (STORE LV) connected to the LV as well as EVs.

The architecture proposed in the SuSTAINABLE project is shown in Figure 4.

\section{5 | Other innovative architectures}

In face of the widespread use of DER in distribution systems, one can argue that a decentralized control architecture capable of solving problems at a local level is more suitable to address the main challenges arising in future power systems.

According to Kariniotakis, Martini, Caerts, Brunner, and Retière (2017), maintaining the current centralized detection and activation paradigm requires significant detailed local information to be collected, aggregated, and communicated from all LV and MV networks to the system operator, which could then detect local problems and determine action to ensure grid secure and optimal reserve activation using LV and MV connected (flexible) resources.

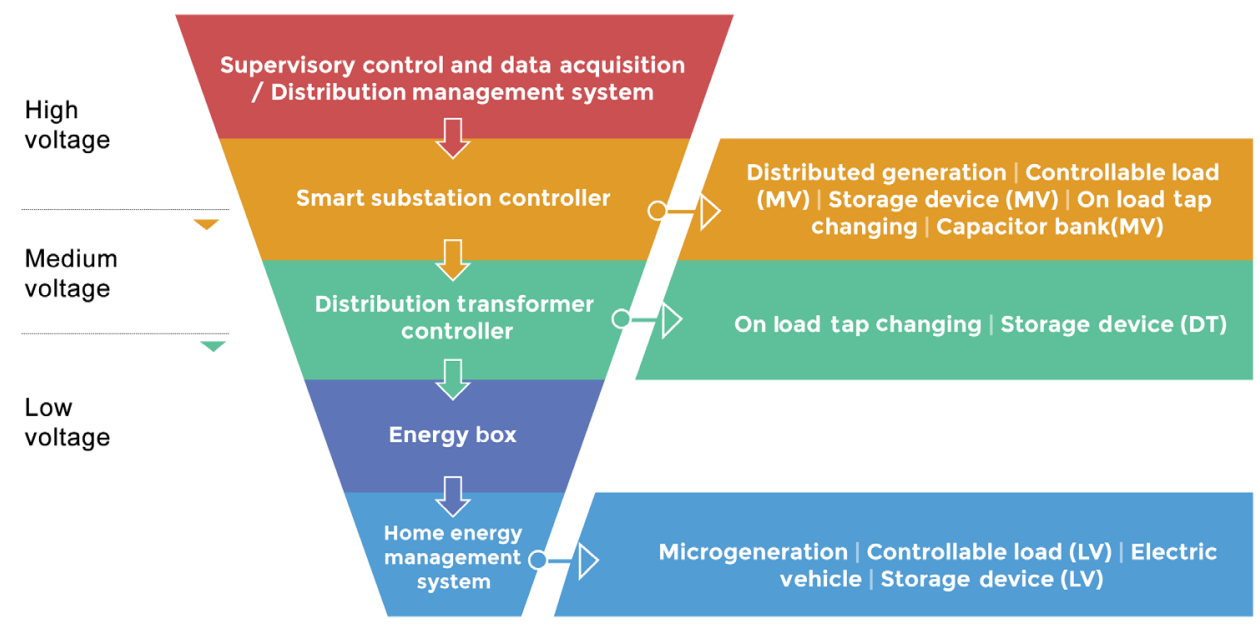

FIGURE 4 Sustainable architecture. (Reprinted with permission from Madureira et al. (2015). Copyright 2015, IET) 
As a result, the FP7 EU project ELECTRA IRP ("European Liaison on Electricity Committed Towards Long-term Research Activity Integrated Research Programme") proposed a decentralized control structure designated Web-ofCells. This approach focuses on addressing tie-line power flow deviations in local intercell (similar to traditional area control error-ACE) instead of system frequency. It is the responsibility of local operators to detect and correct such real-time deviations, which leads to less complexity (in computation) and less communication. A P2P mechanism for intercell coordination is presented to attain a localized imbalance netting result in order to reduce reserve activations in this decentralized paradigm (Kariniotakis et al., 2017).

The project Fractal Grid approach presents a novel paradigm for smart grids using the fractality concept (Kariniotakis et al., 2017). It builds on the hypothesis that the power systems of the future will have a type of fractal organization, based on the recursive assemblage of multiple smart buildings, microgrids, smart cities, distribution grids, national, and international networks. Because Fractal Grids produce more flexible, controllable, resilient, and interoperable power system that supports an efficient and secure operation, the proposed methodology develops an innovative multiscale methodology capable of addressing the increased complexity of future power systems (Kariniotakis et al., 2017).

P2P communication is also being exploited as a reference for future smart grid architectures. In fact, P2P protocols for communication and coordination using gossiping are seen as a way to address scalability and reliability issues in a smart grid (Campos, Matos, Pereira, \& Rua, 2014) in which a framework is proposed that ensures gossip-based dissemination and coordination through web services. This enables using existing standards and still evolves toward a decentralized P2P service architecture that is able to be adjusted according to the requirements of each scenario. (Jogunola et al., 2017) investigated P2P communication architectures for trading and sharing energy from prosumers, including two different protocols, and evaluated their performance through simulation studies. In Zhang, Wu, Zhou, Cheng, and Long (2018), a model for a hierarchical system architecture is presented that is able to identify and categorize the principal elements and technologies involved in P2P energy trading within a microgrid. Moreover, a trading platform for $\mathrm{P} 2 \mathrm{P}$ energy is designed and game theory is used to simulate $\mathrm{P} 2 \mathrm{P}$ energy trading.

\section{I CONCLUSION}

This paper identified the main challenges the electric power systems of the future will face as a result from the simultaneous increase in renewable generation and electrification of the economy, imposed by the need to tackle the climate change threats.

These challenges will lead to the following main changes:

1. An increase in the volume of renewable power generation, connected to the grid through converters based on power electronics, presenting simultaneously a characteristic of time variability in the power output; this also means that potentially millions of generation units connect to the transmission and distribution networks.

2. Consumer participation in grid management, leading to a consumer-centric approach that requires aggregation agents to manage and deal with the capability these consumers may have in providing flexibility, to adjust the consumption to the availability of generation sources and to help manage locally and globally operational constraints.

3. A growing presence of a multilevel energy storage solution, where storage systems of different types will play a key role in providing flexibility, balancing load versus generation and relieving local technical constraints.

The dimension and complexity of such changes in the system will then require the adoption of more decentralized and flexible architectures as well as support by advanced communication infrastructures, to deal with the control, management, and trading needs at the distribution level in an efficient and robust manner. Advanced hierarchical control and management structures will be needed to deal with the "big picture" that regional control centers will need to have for operational management and trading energy and services. This will allow solving in due time problems that are local (namely voltage constraints or power flow branch congestions) and contributing to manage globally problems such as load balancing and frequency control issues.

\section{CONFLICT OF INTEREST}

The authors have declared no conflicts of interest for this article. 


\section{AUTHOR CONTRIBUTIONS}

Joao Abel Peças Lopes: Conceptualization. André Guimarães Madureira: Conceptualization; writing-review, and editing. Manuel Matos: Conceptualization. Ricardo Bessa: Conceptualization. Vítor Monteiro: Conceptualization; writing-review, and editing. João Luiz Afonso: Conceptualization; validation; writing-review and editing. Sérgio Santos: Conceptualization; writing-review and editing. João P. S. Catalão: Conceptualization; writing-review and editing. Carlos Antunes: Conceptualization; writing-original draft, writing-review, and editing. Pedro Magalhães: Conceptualization; writing-review, and editing.

\section{ORCID}

João Abel Peças Lopes (1D) https://orcid.org/0000-0001-7638-1522

\section{ENDNOTES}

${ }^{1}$ Available at: https://www.entsoe.eu/network_codes/rfg/.

${ }^{2}$ Available at: http://www.nicegrid.fr/en/.

\section{RELATED WIRES ARTICLE}

Lopes, J. A. P., Madureira, A. G., \& Moreira, C. C. L. M. (2013). A View of Microgrids. Wiley Interdisciplinary Reviews: Energy and Environment, 2(1), 86-103.

\section{REFERENCES}

Ackermann, T., Prevost, T., Vittal, V., Roscoe, A. J., Matevosyan, J., \& Miller, N. (2017). Paving the way: A future without inertia is closer than you think. IEEE Power and Energy Magazine, 15(6), 61-69.

Bessa, R. J. (2018). Future trends for big data application in power systems. In Big data application in power systems (pp. 223-242). Amsterdam, The Netherlands: Elsevier.

Bollen, M. H., \& Hassan, F. (2011). Integration of distributed generation in the power system (Vol. 80). Hoboken, NJ: John Wiley \& Sons.

Buttler, A., Koltun, R., Wolf, R., \& Spliethoff, H. (2015). A detailed techno-economic analysis of heat integration in high temperature electrolysis for efficient hydrogen production. International Journal of Hydrogen Energy, 40(1), 38-50.

Caire, R., Lisanti, B., \& Vanet, E. (2014). Dream reference object model and dictionary. Deliverable D5.1, EU FP7 Project Distributed Renewable resources Exploitation in electric grids through Advanced heterarchical Management (DREAM). Available from: http://www. dream-smartgrid.eu/downloads/\#deliverables

Campos, F., Matos, M., Pereira, J., \& Rua, D. (2014, September). A peer-to-peer service architecture for the smart grid. In 14-th IEEE international conference on peer-to-peer computing (pp. 1-5). IEEE.

Carrilero, I., Ansean, D., Viera, J. C., Fernandez, Y., Pereirinha, P. G., \& Gonzalez, M. (2017, December). Impact of fast-charging and regenerative braking in LiFePO4 batteries for electric bus applications. In 2017 IEEE vehicle power and propulsion conference (VPPC) (pp. 1-6). IEEE.

Cavalcante, L., Bessa, R. J., Reis, M., \& Browell, J. (2017). LASSO vector autoregression structures for very short-term wind power forecasting. Wind Energy, 20(4), 657-675.

Cazzola, P., Gorner, M., Munuera, L., Schuitmaker, R., \& Maroney, E. (2017). Global EV outlook 2017: Two million and counting. France: International Energy Agency (IEA).

Chandler, H. (2011). Harnessing variable renewables: A guide to the balancing challenge. Paris, France: International Energy Agency.

Christakou, K., Tomozei, D. C., Le Boudec, J. Y., \& Paolone, M. (2017). AC OPF in radial distribution networks-Part I: On the limits of the branch flow convexification and the alternating direction method of multipliers. Electric Power Systems Research, 143, 438-450.

Costa, L. F., De Carne, G., Buticchi, G., \& Liserre, M. (2017). The smart transformer: A solid-state transformer tailored to provide ancillary services to the distribution grid. IEEE Power Electronics Magazine, 4(2), 56-67.

Cunha, L., Reis, J., \& Lopes, J. P. (2008). InovGrid project-distribution network evolution as a decisive answer to new electrical sector challenges. In CIRED Seminar 2008: SmartGrids for Distribution (Vol. 16, pp. 1-2). Frankfurt, Germany: IET.

De Carne, G., Buticchi, G., Zou, Z., \& Liserre, M. (2017). Reverse power flow control in a ST-fed distribution grid. IEEE Transactions on Smart Grid, 9(4), 3811-3819.

Deng, R., Yang, Z., Chow, M. Y., \& Chen, J. (2015). A survey on demand response in smart grids: Mathematical models and approaches. IEEE Transactions on Industrial Informatics, 11(3), 570-582.

Elgowainy, A., Han, J., Poch, L., Wang, M., Vyas, A., Mahalik, M., \& Rousseau, A. (2010). Well-to-wheels analysis of energy use and greenhouse gas emissions of plug-in hybrid electric vehicles (No. ANL/ESD/10-1). Argonne, IL: Argonne National Lab.(ANL).

Eto, J. (1996). The past, present, and future of US utility demand-side management programs (No. LBNL-39931). Berkeley, CA: Lawrence Berkeley National Lab. 
European Commission. (2014). A policy framework for climate and energy in the period from 2020 to 2030. Technical Report COM (2014) 15. Brussels: European Commission.

Foggia, G., Muscholl, M., Passelergue, J. C., Gambier-Morel, P., Vuillecard, C., Krivine, J. P., ... \& Kariniotakis, G. (2014, August). The Nice Grid project: Using distributed energy resources to reduce power demand through advanced network management. In 2014 CIGRE Session. Paris, France: CIGRE.

Garcia, A. A. (1987). Demand side management integration issues a case history. IEEE Transactions on Power Systems, 2(3), 772-778.

Gellings, C. W. (1985). The concept of demand-side management for electric utilities. Proceedings of the IEEE, 73(10), 1468-1470.

Gellings, C. W., \& Smith, W. M. (1989). Integrating demand-side management into utility planning. Proceedings of the IEEE, 77(6), 908-918.

Goli, P., \& Shireen, W. (2014). PV integrated smart charging of PHEVs based on DC link voltage sensing. IEEE Transactions on Smart Grid, 5(3), 1421-1428.

Götz, M., Lefebvre, J., Mörs, F., Koch, A. M., Graf, F., Bajohr, S., ... Kolb, T. (2016). Renewable power-to-gas: A technological and economic review. Renewable Energy, 85, 1371-1390.

Hall, D., \& Lutsey, N. (2018). Effects of battery manufacturing on electric vehicle life-cycle greenhouse gas emissions. Washington DC: International Council on Clean Transportation.

Hamilton, C., Gamboa, G., Elmes, J., Kerley, R., Arias, A., Pepper, M., ... \& Batarseh, I. (2010, November). System architecture of a modular direct-DC PV charging station for plug-in electric vehicles. In IECON 2010-36th annual conference on IEEE industrial electronics society (pp. 2516-2520). IEEE.

Heinen, S., Mancarella, P., O'Dwyer, C., \& O'Malley, M. (2018). Heat electrification: The latest research in Europe. IEEE Power and Energy Magazine, 16(4), 69-78.

Huber, J. E., \& Kolar, J. W. (2017). Applicability of solid-state transformers in today's and future distribution grids. IEEE Transactions on Smart Grid, 10(1), 317-326.

Hughes, T. P. (1993). Networks of power: Electrification in Western society, 1880-1930. London, England: JHU Press.

International Energy Agency. (2015). $\mathrm{CO}_{2}$ emissions from fuel combustion (2015th ed.). Paris, France: Author.

Jogunola, O., Ikpehai, A., Anoh, K., Adebisi, B., Hammoudeh, M., Gacanin, H., \& Harris, G. (2017). Comparative analysis of P2P architectures for energy trading and sharing. Energies, 11(1), 62.

Kariniotakis, G., Martini, L., Caerts, C., Brunner, H., \& Retière, N. (2017). Challenges, innovative architectures and control strategies for future networks: The web-of-cells, fractal grids and other concepts. CIRED-Open Access Proceedings Journal, 2017(1), $2149-2152$.

Khan, M. W., \& Wang, J. (2017). The research on multi-agent system for microgrid control and optimization. Renewable and Sustainable Energy Reviews, 80, 1399-1411.

Khan, M. W., Wang, J., Ma, M., Xiong, L., Li, P., \& Wu, F. (2019). Optimal energy management and control aspects of distributed microgrid using multi-agent systems. Sustainable Cities and Society, 44, 855-870.

Khan, M. W., Wang, J., Xiong, L., \& Ma, M. (2018). Modelling and optimal management of distributed microgrid using multi-agent systems. Sustainable Cities and Society, 41, 154-169.

Khazaei, J., \& Nguyen, D. H. (2017). Multi-agent consensus design for heterogeneous energy storage devices with droop control in smart grids. IEEE Transactions on Smart Grid, 10(2), 1395-1404.

Klaimi, J., Rahim-Amoud, R., Merghem-Boulahia, L., \& Jrad, A. (2018). A novel loss-based energy management approach for smart grids using multi-agent systems and intelligent storage systems. Sustainable Cities and Society, 39, 344-357.

Ko, Y., Chub, A., Costa, L., Andresen, M., \& Liserre, M. (2018, March). Smart transformer universal operation. In 2018 IEEE applied power electronics conference and exposition (APEC) (pp. 1609-1616). IEEE.

Lannez, S., Foggia, G., Muscholl, M., Passelergue, J. C., Lebosse, C., \& Mercier, K. (2013, June). Nice grid: Smart grid pilot demonstrating innovative distribution network operation. In 2013 IEEE grenoble conference (pp. 1-5). IEEE.

Liu, N., Yu, X., Wang, C., Li, C., Ma, L., \& Lei, J. (2017). Energy-sharing model with price-based demand response for microgrids of peer-topeer prosumers. IEEE Transactions on Power Systems, 32(5), 3569-3583.

Lopes, J. A. P., Madureira, A. G., \& Moreira, C. C. L. M. (2013). A view of microgrids. WIREs: Energy and Environment, 2(1), 86-103. https:// doi.org/10.1002/wene. 34

Lotfi, M., Monteiro, C., Shafie-khah, M., \& Catalão, J. P. (2018, December). Evolution of demand response: A historical analysis of legislation and research trends. In 2018 twentieth international middle east power systems conference (MEPCON) (pp. 968-973). IEEE.

Luo, F., Chen, Y., Xu, Z., Liang, G., Zheng, Y., \& Qiu, J. (2016). Multiagent-based cooperative control framework for microgrids' energy imbalance. IEEE Transactions on Industrial Informatics, 13(3), 1046-1056.

Madureira, A., Bessa, R., Seca, L., Pereira, J., Messias, A. A., Lopes, D. A., \& Matos, P. G. (2015). Advanced system architecture and algorithms for smart distribution grids: The SuSTAINABLE approach. In CIRED2015-23rd international conference on electricity distribution.

Matos, M. A., Seca, L., Madureira, A. G., Soares, F. J., Bessa, R. J., Pereira, J., \& Peças Lopes, J. (2016). Control and management architectures. In C.-C. Liu, S. McArthur, \& S.-J. Lee (Eds.), Smart grid handbook (Vol. 2, pp. 539-561). Chichester, England: John Wiley \& Sons, Ltd.

McKenna, E., Richardson, I., \& Thomson, M. (2012). Smart meter data: Balancing consumer privacy concerns with legitimate applications. Energy Policy, 41, 807-814.

Milano, F., Dörfler, F., Hug, G., Hill, D. J., \& Verbič, G. (2018, June). Foundations and challenges of low-inertia systems. In 2018 power systems computation conference (PSCC) (pp. 1-25). IEEE.

Milberg, J., \& Schlenker, A. (2010). Plug into the future. IEEE Power and Energy Magazine, 9(1), 56-65. 
Monteiro, V., Pinto, J. G., \& Afonso, J. L. (2015). Operation modes for the electric vehicle in smart grids and smart homes: Present and proposed modes. IEEE Transactions on Vehicular Technology, 65(3), 1007-1020.

Monteiro, V., Pinto, J. G., \& Afonso, J. L. (2018). Experimental validation of a three-port integrated topology to interface electric vehicles and renewables with the electrical grid. IEEE Transactions on Industrial Informatics, 14(6), 2364-2374.

Moreira, C. L., Resende, F. O., \& Lopes, J. P. (2007). Using low voltage microgrids for service restoration. IEEE Transactions on Power Systems, 22(1), 395-403.

Papaspiliotopoulos, V. A., Korres, G. N., Kleftakis, V. A., \& Hatziargyriou, N. D. (2015). Hardware-in-the-loop design and optimal setting of adaptive protection schemes for distribution systems with distributed generation. IEEE Transactions on Power Delivery, 32(1), 393-400.

Parag, Y., \& Sovacool, B. K. (2016). Electricity market design for the prosumer era. Nature Energy, 1(4), 16032.

Pereirinha, P. G., González, M., Carrilero, I., Anseán, D., Alonso, J., \& Viera, J. C. (2018). Main trends and challenges in road transportation electrification. Transportation Research Procedia, 33, 235-242.

Poudineh, R., Peng, D., \& Mirnezami, S. R. (2017). Electricity networks: Technology, future role and economic incentives for innovation. Oxford, England: Oxford Institute for Energy Studies.

Rokrok, E., Shafie-khah, M., Siano, P., \& Catalão, J. (2017). A decentralized multi-agent-based approach for low voltage microgrid restoration. Energies, 10(10), 1491.

Ruhnau, O., Bannik, S., Otten, S., Praktiknjo, A., \& Robinius, M. (2019). Direct or indirect electrification? A review of heat generation and road transport decarbonisation scenarios for Germany 2050. Energy, 166, 989-999.

Sakurama, K., \& Miura, M. (2017). Distributed constraint optimization on networked multi-agent systems. Applied Mathematics and Computation, 292, 272-281.

Schneider, K. P., Laval, S., Hansen, J., Melton, R. B., Ponder, L., Fox, L., ... Prabakar, K. (2019). A distributed power system control architecture for improved distribution system resiliency. IEEE Access, 7, 9957-9970.

She, X., Huang, A. Q., \& Burgos, R. (2013). Review of solid-state transformer technologies and their application in power distribution systems. IEEE Journal of Emerging and Selected Topics in Power Electronics, 1(3), 186-198.

Shi, C. X., \& Yang, G. H. (2018). Augmented Lagrange algorithms for distributed optimization over multi-agent networks via edge-based method. Automatica, 94, 55-62.

Siano, P. (2014). Demand response and smart grids-A survey. Renewable and Sustainable Energy Reviews, 30, $461-478$.

Smil, V. (2004). World history and energy. Encyclopedia of Energy, 6, 549-561.

Soares, T., Bessa, R. J., Pinson, P., \& Morais, H. (2018). Active distribution grid management based on robust AC optimal power flow. IEEE Transactions on Smart Grid, 9(6), 6229-6241.

Strbac, G. (2008). Demand side management: Benefits and challenges. Energy Policy, 36(12), 4419-4426.

Talari, S., Shafie-Khah, M., Siano, P., Loia, V., Tommasetti, A., \& Catalão, J. (2017). A review of smart cities based on the internet of things concept. Energies, 10(4), 421.

Villar, J., Bessa, R., \& Matos, M. (2018). Flexibility products and markets: Literature review. Electric Power Systems Research, 154, 329-340.

Wu, F. F., \& Varaiya, P. (1999). Coordinated multilateral trades for electric power networks: Theory and implementation. International Journal of Electrical Power \& Energy Systems, 21(2), 75-102.

Zhang, C., Wu, J., Cheng, M., Zhou, Y., \& Long, C. (2016). A bidding system for peer-to-peer energy trading in a grid-connected microgrid. Energy Procedia, 103, 147-152.

Zhang, C., Wu, J., Zhou, Y., Cheng, M., \& Long, C. (2018). Peer-to-peer energy trading in a microgrid. Applied Energy, $220,1-12$.

Zheng, Y., Hill, D. J., \& Dong, Z. Y. (2017). Multi-agent optimal allocation of energy storage systems in distribution systems. IEEE Transactions on Sustainable Energy, 8(4), 1715-1725.

How to cite this article: Lopes JAP, Madureira AG, Matos M, et al. The future of power systems: Challenges, trends, and upcoming paradigms. WIREs Energy Environ. 2019;e368. https://doi.org/10.1002/wene.368 